\title{
The Secret Life of Galaxies
}

\author{
Alan Dressler ${ }^{1}$ and Louis Abramson ${ }^{2}$ \\ ${ }^{1}$ The Observatories of the Carnegie Institution of Science, 813 Santa Barbara Street, \\ Pasadena, CA 91101-1232, USA. email: dressler@obs.carnegiescience.edu \\ ${ }^{2}$ Department of Astronomy \& Astrophysics and Kavli Institute for Cosmological Physics, The \\ University of Chicago, 5640 South Ellis Avenue, Chicago, IL 60637, USA. email: \\ labramson@uchicago.edu>
}

\begin{abstract}
We have learned much about galaxy evolution since $\mathrm{z}=2$, and something to even higher redshifts. How can it be that we know so little about the star formation histories (SFHs) of individual galaxies? Although great progress has been made accumulating huge samples with only rudimentary properties, progress in galaxy evolution means connecting what we've learned to detailed measurements of the life-histories of specific — not just representative - systems.
\end{abstract}

Keywords. galaxies: evolution - galaxies: formation

\section{Introduction}

After a decade of scatter diagrams showing a point for each of hundreds of thousands of galaxies, each measured in some simple way that belies the complexity of the complicated life-histories that each galaxy, there is renewed interest in connecting the detailed knowledge we have about the Milky Way and its neighbors with the billions of galaxies we see at past epochs, almost to their very beginnings. In this paper we present examples of this shift from the general to the particular that promise to move the field forward.

\section{The Green Valley is Purple}

The Sloan Digital Sky Survey (SDSS) provided, for the first time, photometry for hundreds of thousands of nearby galaxies. Among the first remarkable results in galaxy evolution to be addressed with this enormous data sample was the issue of star forming versus non-starforming - passive — galaxies. Kauffman et al. (2003) made a significant step by plotting the star formation rate (SFR), as measured by the broad-band index D4000 versus the galaxy mass, derived by fitting stellar population models to spectral energy distributions (SEDs) from SDSS multicolor photometry. Traditionally, galaxy color, used as a proxy for star formation rate, had been plotted against galaxy luminosity to show the well-known bimodality of red vs. blue galaxies first described by Baade (1958). Kauffman et al. showed something new, that galaxies in the contemporary universe are bimodal — star forming or passive — only if they have masses $\lesssim 10^{11} \mathrm{M}_{\odot}$. Above this mass, nearly all are passive.

The richly populated plot of D4000 vs stellar mass also showed a smaller population in between the passive "red-sequence" and the star forming "blue cloud." Beginning with Faber et al. (2005), dozens of studies identified these inhabitants of the "green valley" $(\mathrm{GV})$ as "quenched galaxies" - those making their way from the blue cloud to the red sequence as the result of a generic event that truncated star formation.

Recently, Schwanski et al. (2014) have showed that ultraviolet colors of GV galaxies do not support the idea of a in-process transformation. Here we present other evidence 

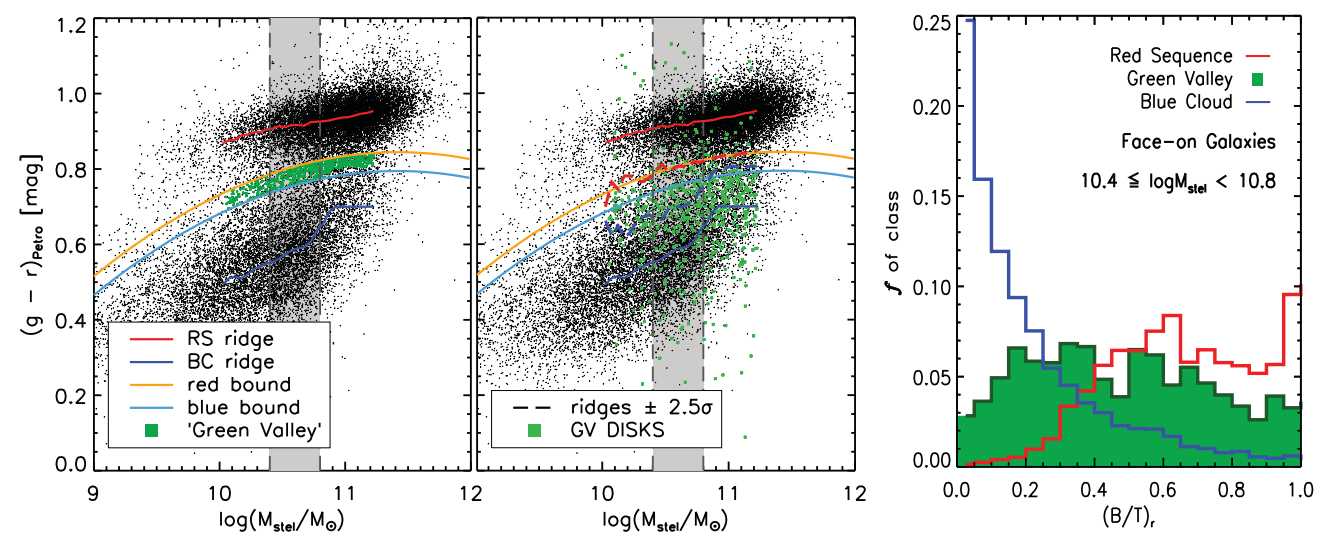

Figure 1. The left panel plots g-r color versus stellar mass, for the 'face-on' SDSS sample, showing the blue cloud and red sequence, and the 'Green Valley' (GV). The right panel shows the bulge-to-total light for these three distributions, limited to galaxies of mass $10.5<\log$ $\mathrm{M}_{\text {stellar }}<10.8$, comparable to the Milky Way. Unlike the largely small-bulge galaxies of the blue cloud, most GV galaxies have substantial bulges, substantially explaining their intermediate color without a quenching process. The center panel repeats the left panel but with the bulges subtracted from the GV galaxies, showing that their starforming disks are those of normal starforming galaxies.

that this often-cited but unproven idea of 'galaxies in transition' is incorrect. Our hypothesis is that the primary reason galaxies appear in the GV is because they have normal star-forming disks but also substantial bulges which — viewed together - produce the intermediate color. This idea emerged from a study of the star forming main sequence (discussed below) by Abramson et al. (2014), which showed that taking the nonstarforming bulges properly into account can resolve the puzzling feature of a "break" in this relation at higher masses - galaxies that are, in fact bulge dominated.

Returning to the GV, we also recognize that many of its inhabitants may simply be dusty, edge-on blue-cloud galaxies, so we remove them from our test sample by keeping only 'face-on' galaxies (ellipticity < 0.2). In Figure 1 (left) we plot $(\mathrm{g}-\mathrm{r}$ ) color as a function of stellar mass for an SDSS sample with $z<0.20$. We define the GV by the orange and blue lines, and, after excluding these points, define the red sequence and blue cloud - traced by the median lines. We take our measurements of bulge-to-total (r-band) light (B/T) from Simard et al. (2011). In Figure 1 (right), we plot the B/T distributions for galaxies of the red-sequence, the blue-cloud, and the GV for the mass limited sample indicated. As expected, the blue cloud galaxies are disk-dominated, the red sequence galaxies bulge-dominated, but the GV galaxies are intermediate, suggesting that, if they were "quenched" blue-cloud galaxies, strong bulge growth would have been required. We prefer the simpler explanation, that the vast majority of these GV galaxies are not in transition, but simply the combination of red bulges and blue disks. We demonstrate this in Figure 1 (center), in which the green points now show the GV galaxy colors after removing their bulges. - the disks of GV galaxies have normal blue-cloud colors.

The idea that most GV galaxies are "recently quenched and transiting" to the red sequence is shown to be incorrect by these data. However, it is reasonable to ask what fraction of the GV galaxies could have been recently quenched. Figure 2 (left) plots Lickindex $\mathrm{H} \delta$ absorption-line strengths versus star formation rate, from $\mathrm{H} \alpha$ measurements of SDSS fiber spectra, for the Figure 1 sample. There is a well- known relation of increasing $\mathrm{H} \delta$ absorption-line strength with SFR that is due to increasing fractions of hot, young stars. The scatter relates mostly to dust in these galaxies. The presence of strong $\mathrm{H} \delta$ 

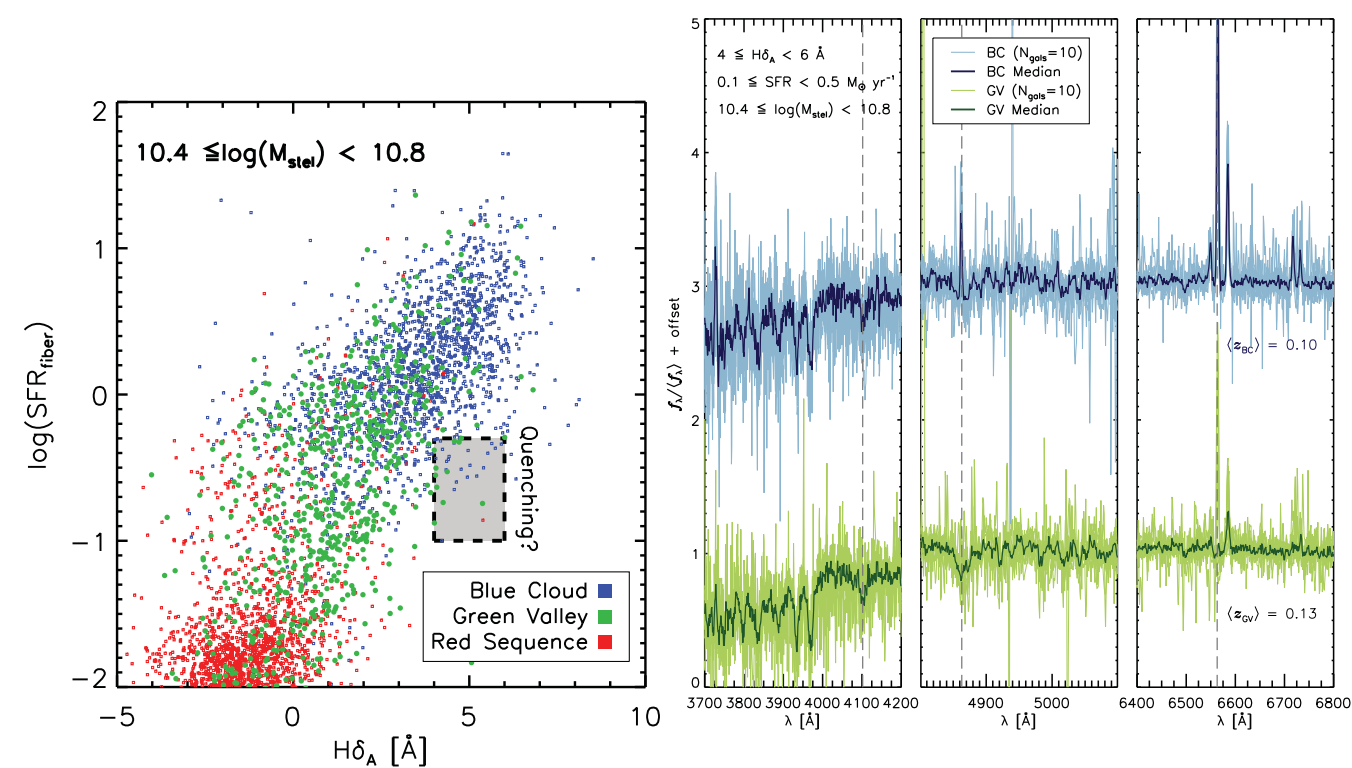

Figure 2. (left) The relationship of SFR and $\mathrm{H} \delta$ absorption for the $10.5<\log \mathrm{M}_{\text {stellar }}<10.8$ subsample of Figure 1 The grey box contains galaxies with strong- $\mathrm{H} \delta$ that are candidates for rapid quenching of star formation. (right) The composite spectra of blue cloud and GV galaxies in the grey box confirm that the latter matches the former except for a recent truncation of star formation, consistent with the idea that these galaxies are rapidly transitioning from the blue cloud to the red sequence. Quenched galaxies represent only a few percent of the GV galaxies in the SDSS sample.

in-excess of this relation is a signature of truncated star formation, or starbursts (e.g., Poggianti et al. 1999). In this diagram the GV galaxies generally track the blue-cloud galaxies, exhibiting the 1:10 ratio of these two populations. However, on the high- $\mathrm{H} \delta$ edge of this relation we see a proportion $\sim 1: 1$, meaning that there are in fact a larger fraction of $\mathrm{H} \delta$-strong spectra. We select blue could and GV galaxies from a portion of this distribution, shown as the grey box in Figure 2 (left), and show the composite spectra of $10 \mathrm{GV}$ and 10 blue-cloud galaxies in Figure 2 (right). Here, indeed, we see evidence of a quenched population. The color and absorption-line-strengths of the blue-could and GV composite are an excellent match, but the former has emission lines - most notably, $\mathrm{H} \alpha$ and $\mathrm{H} \beta$ - that are weak or absent in latter. This is exactly what is expected for quenched systems, where the 1-10 Myr-old stars that power the H II regions have disappeared, but the stellar population $\tau>100$ Myr remains unchanged. Over a timescale $\tau \sim 1$ Gyr, these GV galaxies should join the red sequence.

We estimate that, for the SDSS sample, the size of this quenched population is a few percent of the starforming population. In her talk at this Symposium, Professor Sandra Faber referred to these cases as "fast-track" quenching. She acknowledged that most galaxies in the GV are following the "slow-track," those with a time scale of several Gyr - indistinguishable from normal galaxy evolution, and not the result of a "quenching event." We conclude that for present-epoch galaxies the GV is basically composed of intermediate-bulge spiral galaxies that are slowly, steadily making their way from the blue cloud to the red sequence. through the normal process of gas exhaustion. Vulcani et al. (2014) come to similar conclusions in their study comparing the colors and morphologies of PM2GC galaxies $(z \sim 0.1)$ in the $\mathrm{GV}$, red sequence, and blue cloud. counterparts. 
Professor Faber also suggested that "fast-track" quenching might be a larger component of early universe galaxies. We note that the situation at intermediate-redshift is similar to what we have discussed here, with an additional ten-times larges population of starburst and poststarburst galaxies (e.g., Dressler et al. 2013). One might think that these are cases in which star formation has been stopped - by means of a starburst, but in Dressler et al. and Abramson et al. (2013) we provide strong evidence that most starbursts do not transform blue cloud galaxies to red. If they did, the number of intermediate-z galaxies $\left(\log \mathrm{M}_{\text {stellar }}>10.2\right)$ on the red sequence would be growing much faster than observed. Rather, we propose that these starbursts are the result of minor mergers, and that when they occur in star forming galaxies, they return to same. Likewise, when a red-sequence galaxy suffers a minor merger, it generally returns to its passive state. We think that only a major merger is likely to lead to a starbust-mediated transformation from a starforming to passive galaxy.

\section{A Better Parameterization of Star Formation Histories?}

The IMACS Cluster Building Survey team, including the authors of this paper plus Gus Oemler, Mike Gladders, Bianca Poggianti, and Benedetta Vulcani, is pursuing the question of the possible forms of the SFHs of galaxies. A common approach to this issue is to consider the 'Star Forming Main Sequence' (SFMS: Noeski et al. 2007; Whitaker et al. 2012), a plot of an evolving relation of SFR and stellar mass, as a template for galaxy evolution (e.g. Peng et al. 2010). If the scatter were small - possibly consistent with observational errors, as suggested in these studies - it might be possible to reduce SFHs to a single path, one that connects the SFR uniquely to a mass at any given epoch. On the other hand, if the scatter is real, then "connecting the dots" from the SFMS of one epoch to another is a difficult if not hopeless task.

The ICBS team has assembled evidence that the scatter in the SFMS is real, and large - approaching 0.5 dex if the sample is not "pruned" and when selection effects are properly taken into account (Oemler et al. 2014; see also Salim et al. 2007). We believe this is strong evidence for diverse SFHs for galaxies at any mass. Oemler et al. (2013) compared the distribution of specific star formation rates, sSFRs, of ICBS field galaxies to present-epoch field galaxies and found that, at $z=0.4,20 \%$ of all galaxies could not be fit by traditional $\tau$-models, that is, they exhibit rising star formation rates at that epoch. Searching for an analytical form that can fully fit such diversity in SFHs, we noted, as described in Gladders et al. (2013), that the Madau plot — the SFH of the universe itself - is well fit by a lognormal. We show this here using the new compilation of star formation rate densities from Madau and Dickinson (2014) in Figure 3 (left), where the authors have included a polynomial fit, which we imagine as physically unmotivated. An arguably better fit is achieved by the lognormal, a simple form that occurs frequently in natural systems and is described by only two, easily understandable parameters, in this case $\mathrm{t}_{0}$ - the time of peak star formation, and $\tau$ - the half-width of the SFH.

If the lognormal is a good fit to the universal SFH, might it be a good description of individual SFHs as well? Gladders et al. (2013) tested that supposition by using as constraints the $\mathrm{SSFR}$ distribution composed of two $z \approx 0$ samples and the Madau plot itself, and requiring each of the present-epoch galaxies to have a SFH that is lognormal in time. The model, constrained only by the local sample and the Madau relation, correctly predicts the distribution of SSFR for the ICBS and AEGIS samples at $z=0.2,0.4,0.6$, and 0.8. (Gladders et al. — see Figure 7), in contrast to an attempt using Gaussian SFHs that fails badly (ibid, Figure 12). Adding in the sSFHs for the intermediate-redshift data produces modestly better constraints: Figure 3 (right) shows the distribution of $\left(\mathrm{t}_{0}, \tau\right)$ 

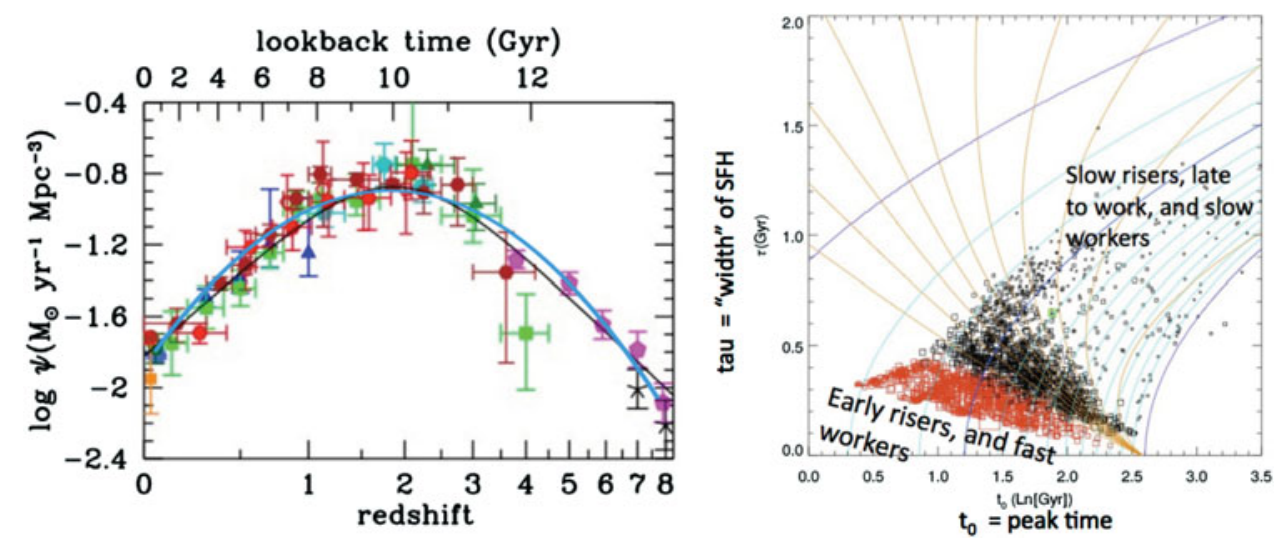

Figure 3. (left) The "Madau diagram," from Madau and Dickinson (2014). The thick blue line is a lognormal fit with $\mathrm{t}_{0}=4.66 \mathrm{Gyr}$ and $\tau=1.78 \mathrm{Gyr}$, which is arguably a better fit to the data than the polynomial constructed by those authors. (right) The distribution of $\left(\mathrm{t}_{0}, \tau\right)$ parameters for the lognormals describing the evolution of the present-epoch field sample. Passive galaxies, defined by Gladders et al. as SFR $<0.05 \mathrm{M}_{\odot} \mathrm{yr}^{-1}$ form the sequence of red points, while the starforming galaxies scatter, exhibiting a wide range of SFHs.

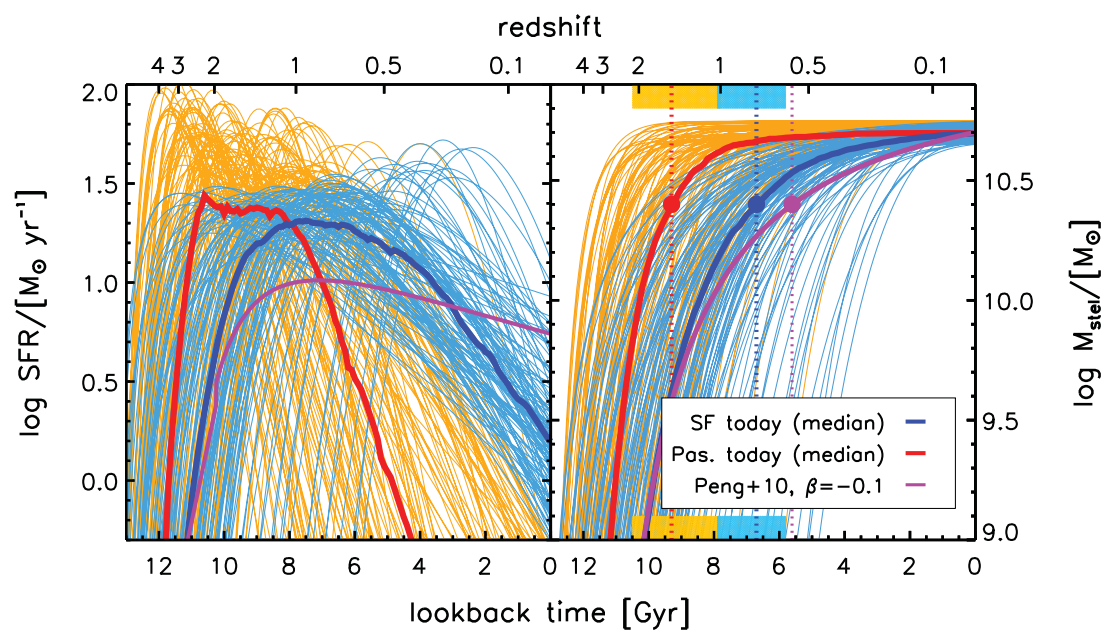

Figure 4. (left) Lognormal SFHs (left) and mass-growth curves (right) for today's Milky Waymass galaxies, from the model by Gladders et al. (2013). Thin orange/blue lines, respectively, denote currently "passive" ( $\log \mathrm{sSFR} / \mathrm{yr}^{-1} \leqslant-11$ ) or "starforming" (sSFR above that threshold) galaxies. Thick red/blue tracks show median $\operatorname{SFR}(t)$ for each class. While, on average, these populations had very different SFHs, our modeling predicts a wide diversity of growth histories within each class (see vertical lines/banding at right denoting their median/inter-quartile spread in half-mass times). Such diversity is not a feature of models such as that of Peng et al. (2010) - which yield a single track for all starforming objects at fixed stellar mass, today (pink curves) - and should be testable with advanced methods of stellar-population synthesis.

for this final fit of Gladders et al. As we will show in an upcoming paper (Abramson et al. 2014), using only these $z<1$ data we can fit the state-of-the-art mass functions of Tomczak et al. (2014) of both passive and star forming galaxies for $0.5<z<2.5$, even though mass was never used to inform the SFH modeling.

In summary, a model including a wide diversity of lognormal SFHs reproduces today's three common relations that record the evolution of basic galaxy parameters over cosmic time: the Madau plot of SFR-density; the SFMS with scatter; and the mass functions 
of both starforming and passive galaxies. However, our success is not unique, as Peng et al., Kelson (2014), and Behroozi and collaborators (Behroozi et al. 2013a,b) have all claimed the same for very different descriptions of SFHs (see also Simha et al. 2014). The galaxies in these studies are represented as numbers - measures of basic or even integral properties, for points that cannot in general be connected over time. It is not surprising, then, that the Madau diagram, SFMS, and mass functions are insufficient to choose between different models of SFHs. Our formalism allows for much more diversity than simply "starforming" or "quenched." Figure 4 (left) is a plot showing the diversity of our lognormal SFHs, both as aggregate SFHs for star forming galaxies contrasted to today's passive galaxies, and for individual SFHs. Also plotted is the single SFH of a galaxy that is star forming today in the Peng et al. model. Figure 4 (right) shows the mass growth derived for these SFHs. Even in the average of lognormal histories, their is substantial difference in galaxy growth over time - the SFHs for galaxies at the same mass can be very diverse and still fit available data.

The challenge is clear: we need to move beyond these three basic parameterizations and test the SFHs of individual galaxies, as we have begun to do with the Milky Way and its neighbors. Only when such detailed measurements replace the general, population-based data we have today will the full picture of galaxy star formation histories emerge.

\section{Acknowledgements}

The authors thank the ICBS team for many intense and inspiring discussions.

\section{References}

Abramson, L. E., Dressler, A., Gladders, M. D., et al. 2013, ApJ, 777, 124

Abramson, L. E., Gladders, M. G., \& Dressler, A., et al. 2014, in preparation.

Baade, W. 1958, Ricerche Astronomiche, 5, 3

Behroozi, P. S., Wechsler, R. H., \& Conroy, C. 2013a, ApJ, 770, 57

Behroozi, P. S., Marchesini, D., Wechsler, R. H., et al. 2013b, ApJ, 777, L10

Dressler, A., Oemler, A., Jr., Poggianti, B. M., et al. 2013, ApJ, 770, 62

Gladders, M. D., Oemler, A., Dressler, A., et al. 2013, ApJ, 770, 64

Kelson, D. D. 2014, arXiv:1406.5191

Noeske, K. G., Weiner, B. J., Faber, S. M., et al. 2007, ApJ, 660, L43

Oemler, A., Jr., Dressler, A., Gladders, M. G., et al. 2013, ApJ, 770, 63

Oemler, A., Jr., Abramson, L. E., Gladders, M. G., et al. 2014, in preparation.

Madau, P. \& Dickinson, M. 2014, ARA\&A, 52, 415

Peng, Y.-j., Lilly, S. J., Kovač, K., et al. 2010, ApJ, 721, 193

Poggianti, B. M., Smail, I., Dressler, A., et al. 1999, ApJ, 518, 576

Simard, L., Mendel, J. T., Patton, D. R., Ellison, S. L., \& McConnachie, A. W. 2011, ApJS, 196,11

Schawinski, K., Urry, C. M., Simmons, B. D., et al. 2014, MNRAS, 440, 889

Simha, V., Weinberg, D. H., Conroy, C., et al. 2014, arXiv:1404.0402

Tomczak, A. R., Quadri, R. F., Tran, K.-V. H., et al. 2014, ApJ, 783, 85

Vulcani, B., Poggianti, B. M., Fritz, J., et al. 2014, submitted to ApJ. Abramson, L. E., Gladders, M. G., et al. 2014, in preparation.

Whitaker, K. E., van Dokkum, P. G., Brammer, G., \& Franx, M. 2012, ApJ, 754, L29 